\title{
The Electric Resistance of Shape Memory Alloys in the Pseudoelastic Regime
}

\author{
G. Airoldi****, D.A. Lodi*, $\left.{ }^{1}\right)$ and M. Pozzi**** \\ * Dipartimento di Fisica, Università di Milano, via Celoria 16, 20133 Milano, Italy \\ ** Istituto Nazionale per la Fisica della Materia, Unità di Milano Università, via Celoria 16, \\ 20133 Milano, Italy
}

\begin{abstract}
The electric resistance, sensitively dependent on the electronic structure and currently used to detect transformation temperatures in shape memory alloys, deserves attention also in the stress transformation domain. In fact, the electric resistance has already been investigated under increasing stress in the martensitic phase of several shape memory alloys: in this case a linear relationship is found between the electric resistance variation and the built-in deformation related to the variant reorientation process, at least in NiTi alloys. In the stress transformation range at constant temperature, the results are less clear, notably in NiTi alloys where two transformations, the Rphase $\rightarrow M$ and the $P \rightarrow M$, are often present. The aim of this paper is to investigate the electric resistance dependence in the pseudoelastic regime in some selected shape memory alloys where one single transformation is present. Attention has been focused here on a $\mathrm{Ni}_{25} \mathrm{Ti}_{50} \mathrm{Cu}_{25}$ alloy, obtained by melt-spinning, where just the cubic(B2)↔orthorhombic(B19) transformation is present and on a CuAlBe alloy where the cubic $\left(\mathrm{DO}_{3}\right) \leftrightarrow$ orthorhombic $(18 \mathrm{R})$ is expected. Both alloys show good pseudoelastic loops though with different features: the former shows a critical stress to induce the B19 phase constant all along the transformation plateau, whilst the latter shows a linear superelastic behaviour. The electric resistance variation, detected during the stress induced martensitic transformation, shows a nice linear behaviour, reversible with the transformation direction. The results are compared with the ones obtained on NiTi alloys.
\end{abstract}

\section{INTRODUCTION}

The electric resistance (ER) of shape memory alloys (SMAs) has always been used, in the temperature domain, to detect the transformation temperatures of the thermoelastic martensitic transformation exploiting the resistivity change due to the electronic structure modification. Widespread results have appeared in the literature connected with the cubic B2 to monoclinic B19'-structure (as in NiTi[1], NiTiCu ${ }_{5}$ [2] alloys) or with the cubic B2 to orthorhombic B19 transformation (as in $\mathrm{Ni}_{40} \mathrm{Ti}_{50} \mathrm{Cu}_{10}$ alloys [3-5]); in some cases, as in NiTi alloys, where two transformations may be present (the B2 $\rightarrow$ Rphase and the Rphase $\rightarrow B 19^{\prime}[6]$ ), even overlapping in the same temperature domain, the sign of the resistivity change connected with the electronic structure modification has greatly helped to identify the sequence of transformations on the temperature scale.

The results obtained at constant temperature in the stress-strain domain, in the martensitic state, have, on the other hand, suggested $[7,8]$ that the electronic contribution can be related to the variants oriented by the stress state.

In the stress induced martensite transformation range, where the martensite variant with the lowest Schmidt factor first grows under an applied stress, the electronic contribution connected with the martensite fraction can add to, or contrast the contribution connected with deformation.

Since a clear-cut view of the ER as a function of strain is however lacking in the literature, in the stress induced martensite (SIM) regime, investigations of different alloy systems permitting to examine the relevance of the sign of the electronic contribution have stimulated the present results.

(') Now at: SGS-Thomson, via Olivetti, Agrate Brianza, Milano, Italy 


\section{EXPERIMENTAL}

\subsection{Materials}

$\mathrm{Ni}_{25} \mathbf{T i}_{50} \mathrm{Cu}_{25}$ ribbons obtained by melt spinning (width $=1.76 \pm 0.02 \mathrm{~mm}$; thickness $=32.7 \pm 1.3 \mu \mathrm{m}$ ), with typical specimen gauge lengths $\approx 120 \mathrm{~mm}$, were used. The specimens, amorphous in the as-spun state [9], were subjected to a thermal treatment at $500{ }^{\circ} \mathrm{C}$ for $300 \mathrm{~s}$ in air, in order to reach recrystallization $\left(\mathrm{A}_{\mathrm{f}} \approx 70^{\circ} \mathrm{C}\right)$.

CuAlBe wires, $800 \mu \mathrm{m}$ in diameter, $\approx 160 \mathrm{~mm}$ in length, supplied by Tréfimétaux, were examined in the as-received state $\left(A_{f} \approx-15^{\circ} \mathrm{C}\right)$.

Commercial NiTi thin wires, supplied by Dinalloy (USA), $54.2 \pm 0.2 \mu \mathrm{m}$ in diameter and $\approx 150 \mathrm{~mm}$ in length were used in the as-received state $\left(A_{f} \approx 70^{\circ} \mathrm{C}\right)$.

\subsection{Procedures}

Tensile stress was applied by a Zwick 1445 testing machine (load cell $100 \mathrm{~N}$ or $500 \mathrm{~N}$, depending on the specimen) at constant crosshead speed $(0.3$ or $0.5 \mathrm{~mm} / \mathrm{min}$ ). The specimen elongation was measured via the crosshead travel monitor with $1 \mu \mathrm{m}$ resolution.

The ER was synchronously detected during the mechanical tests, by the conventional four terminals DC method, using a microohmmeter Burster Resistomat type 2305 (with resolution $10^{-4}$ ).

The test temperature ranged from $-20^{\circ} \mathrm{C}$ to $90^{\circ} \mathrm{C}$ : during isothermal tests the temperature (T) was kept constant within $\pm 0.5^{\circ} \mathrm{C}$ using a circulating liquid thermostatic chamber equipped with a Lauda RC20 thermostat.

The normalised ER/ER 0 vs. T curves, where $\mathrm{ER}_{0}$ is the specimen resistance at high temperature in the parent phase, for typical specimens of the $\mathrm{Ni}_{25} \mathrm{Ti}_{50} \mathrm{Cu}_{25}, \mathrm{CuAlBe}$ and NiTi alloys here examined, are plotted respectively in Figure 1.a,b and c across the transformation range from high temperature parent $(\mathrm{P})$ phase to non oriented martensite $\left(\mathrm{M}_{\mathrm{no}}\right)$. The empty circles on the curves represent the test temperatures adopted to investigate the ER vs. strain

\section{RESULTS}

\section{$\mathrm{Ni}_{25} \mathrm{Ti}_{50} \mathrm{Cu}_{25}$ alloy}

It is found from figure 1.a that the low-temperature phase (B19) has a larger resistivity than the hightemperature phase (B2). Extrapolating the ER of the B19 phase to higher temperatures, a rise between 11 and $12 \%$ in the ER is expected at the $\mathrm{P} \rightarrow \mathrm{M}_{\mathrm{no}}$. transformation.
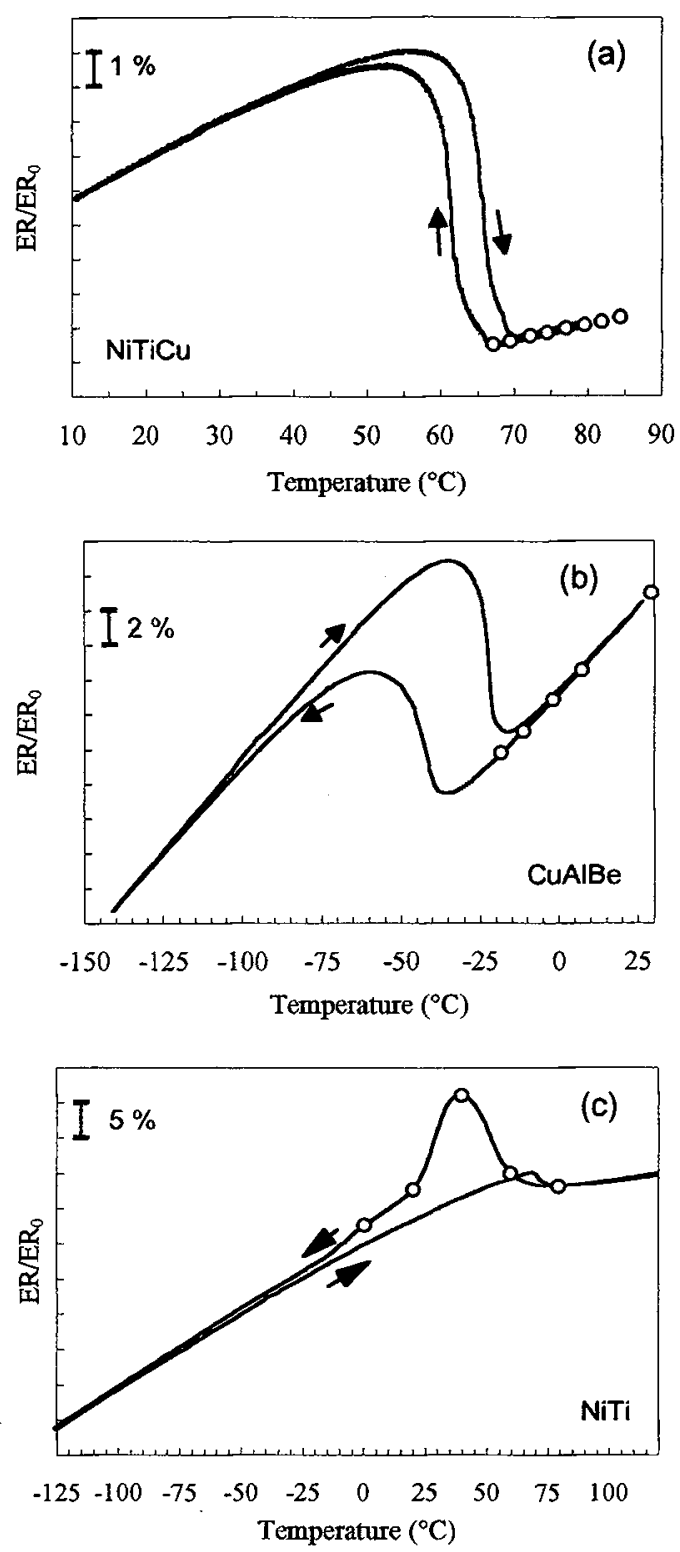

Figure 1. Normalised electric resistance vs. temperature for the three examined alloys: (a) $\mathrm{Ni}_{25} \mathrm{Ti}_{50} \mathrm{Cu}_{25}$, (b) CuAlBe, (c) NiTi. 

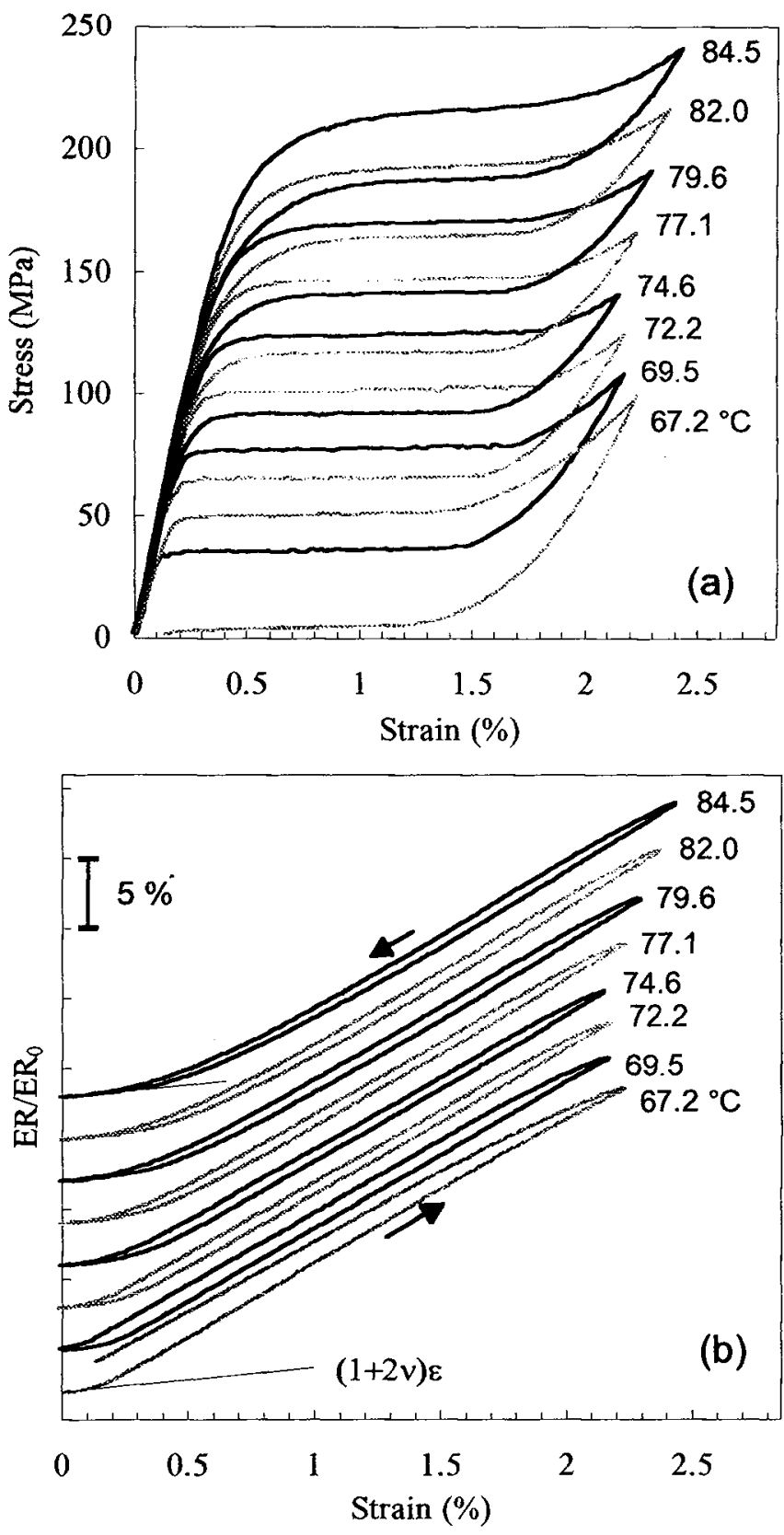

Figure 2. Tensile tests on a NiTiCu specimen: (a) stress vs. strain (b) ER vs. strain. The figures on the plot represent the test temperatures. For clarity, the ER curves are shifted along the $y$-axis.
Figure 2.a shows the stress $(\sigma)$ vs. $\operatorname{strain}(\varepsilon)$ curves, obtained during the isothermal tests. The curves show an initial 'elastic' range, which grows in extension with temperature, followed by a smooth plateau, with a very nice pseudoelastic behaviour. On unloading, the reversion plateau is shifted approximately $0.08 \%$ towards smaller deformation values, as compared to the direct one. As expected from figure $1 . a$, the deformation applied at $67.2^{\circ} \mathrm{C}$ is not completely recovered on mere unloading.

The ER/ER, with $\mathrm{ER}_{0}=\mathrm{ER}(\varepsilon=0)$, is plotted in figure $2 . \mathrm{b}$ as a function of $\varepsilon$, and was detected at the same time as the stress in figure 2.a: after a low slope range, confined to $\varepsilon<0.4 \%$ and associated to the 'elastic' deformation of the parent phase, a high slope linear growth sets in at the stress induced transformation from $\mathbf{P}$ to oriented martensite $\left(\mathrm{M}_{\mathrm{or}}\right)$. Another interesting feature of these curves is the small hysteresis shown: on unloading the ER is larger, for the same deformation values, than that on loading. The hysteresis width is estimated to be around $0.09 \%$ on the deformation scale, and can be explained by the previously mentioned shift between the two plateaus. Thus the ER appears a more reliable state variable than the deformation, in the sense that it is more sensitive to the M-P ratio, while the deformation suffers from differences in the stress state.

As for the ER vs. $\varepsilon$ curve at $67.2^{\circ} \mathrm{C}$, a larger hysteresis is found: after unloading a small pseudoplastic deformation is left, connected with the thermally stable martensite. The higher ER value found at the end of unloading is in agreement with this view.

\section{CuAlBe alloy}

In the $\mathrm{CuAlBe}$ case (figure 1.b), the $\mathrm{P} \rightarrow \mathrm{M}_{\text {n.o. }}$ transformation induces $\mathrm{a} \approx 17 \%$ rise in the ER. Figure 3.a shows the $\sigma-\varepsilon$ curves obtained in isothermal condition: a superelastic rather than pseudoelastic behaviour 
is seen, since there is no well defined plateau. Full superelastic behaviour is found just for $\mathrm{T}=29.3^{\circ} \mathrm{C}$, though $\mathrm{A}_{\mathrm{f}}$ is as low as $-13^{\circ} \mathrm{C}$. At lower temperatures some pseudoplastic deformation is left after unloading, which is fully recovered by heating to room temperature. Figure 3.b shows the normalised ER vs. $\varepsilon$ curves, detected at the same time as the $\sigma-\varepsilon$ curves in figure 3.a. At $29.3^{\circ} \mathrm{C}$ the loading and unloading paths are indistinguishable, whilst they become more and more separated when the temperature is lowered.

\section{NiTi alloy}

Measurements on a NiTi alloy were also performed. Figure 1.c shows three phases on cooling (B2, Rphase, B19') and essentially two on heating from low temperatures (B19', B2). The test temperatures were selected in order to have either pseudoelastic behaviour or thermally stable SIM with different Rphase fractions at the start. At high temperatures, 79.7 and $60.1^{\circ} \mathrm{C}$, the specimen shows a pseudoelastic behaviour (figure 4.a), whilst at lower temperatures the SIM is thermally stable, a pseudoplastic deformation is left after unloading and its complete recovery is attained by heating to parent phase. After an initial 'elastic' range, a plateau follows, which is rough at high temperatures and very smooth at low. The ER vs. $\varepsilon$ curves are given in figure 4.b: the most striking feature is the strong dependence of the overall slope upon temperature.

\section{DISCUSSION}

In the pseudoelastic regime, if only two phases are found, the normalised ER can be written:

$$
\frac{\mathrm{ER}(\varepsilon)}{\mathrm{ER}_{0}}=(1+2 v) \varepsilon+\beta \varepsilon+\cos \mathrm{t}
$$

where $v$ is the Poisson ratio of the process and $\beta$ is a coefficient which takes into account the electronic contribution of the transformation from parent phase to oriented martensite $\left(\mathrm{M}_{\mathrm{or}}\right)$. Equation (1) is obtained
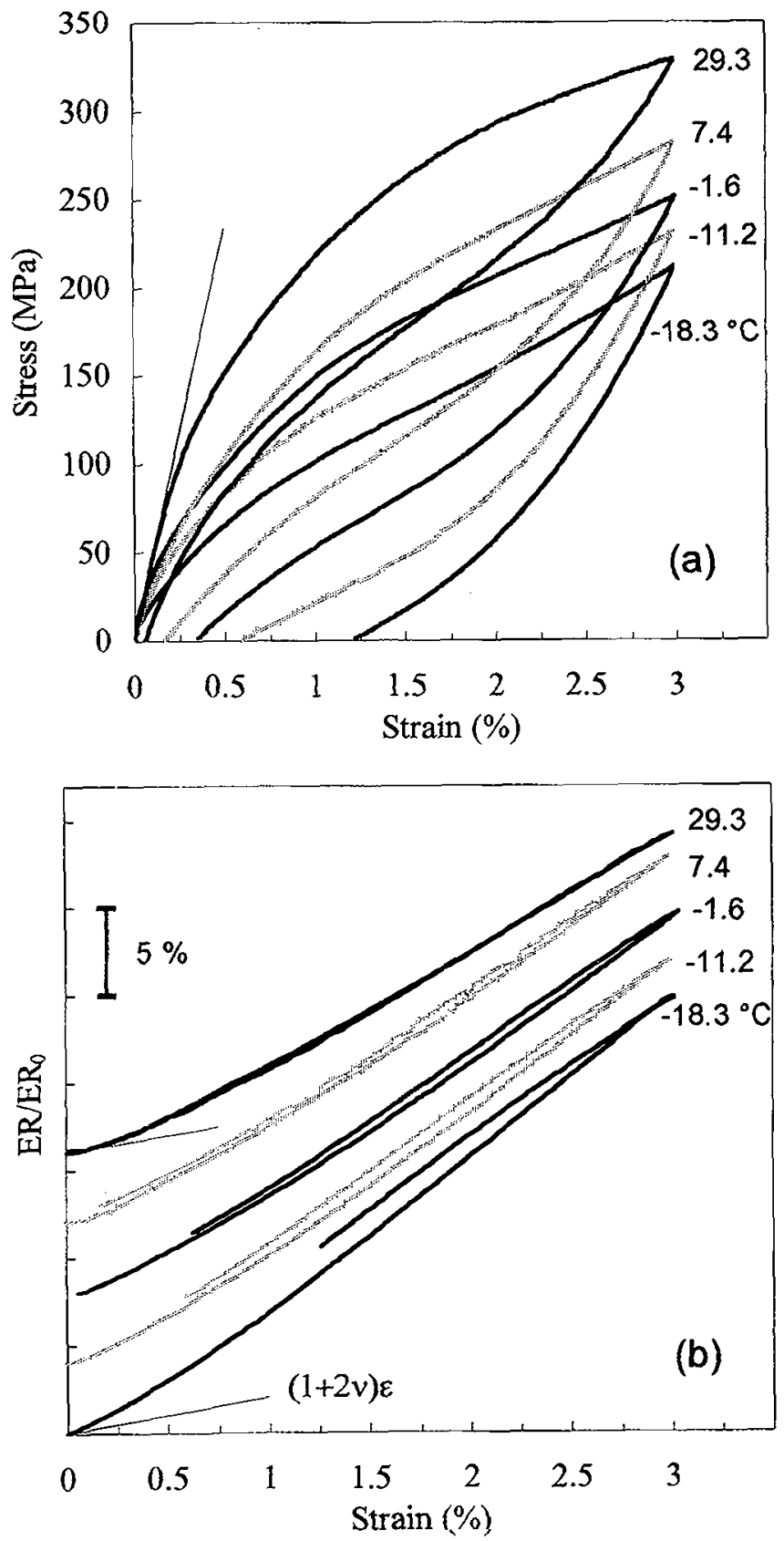

Figure 3. Tensile tests on a CuAlBe alloy: (a) stress vs. strain (b) ER vs. strain. The figures on the plot represent the test temperatures. For clarity, the ER curves are shifted along the y-axis. 
on the assumption that the martensite amount grows linearly with deformation and that the two phases can be considered, from an electric point of view, to be placed in series, so that the total ER is a linear combination of the contribution of the two phases. In deriving this equation, the initial 'elastic' range and the shift between the two plateaus were not taken into account. The existence of this shift means that the measured deformation differs from the transformation deformation, because the former contains the elastic contribution which changes with the value of the transformation stress.

When applied to the curves on NiTiCu ribbons (figure 2.b), with $v$ assumed equal to 0.35 , equation (1) gives $\beta \approx 8.4$. When stress inducing the martensite, the two processes of $\mathbf{P} \rightarrow \mathbf{M}_{\text {no. }}$ transformation and of martensite reorientation occur simultaneously; therefore the two contributions to the electric resistivity are expected to add to a total variation of ER when creating SIM. Actually, the two contributions can be evaluated separately: the former from ER vs. $T$ measurements (figure 1.a), the latter from tensile tests in the martensite state [10]. In fact, $\beta$ has a figure of 8.4 which is approximately equal to the sum of the $\mathrm{P} \rightarrow \mathrm{M}_{\text {no. }}$ contribution deduced from figure 1.a $\left(\left(\Delta \mathrm{ER} / \mathrm{ER}_{0}\right) / \Delta \varepsilon \approx 11 \% / 2.2 \%=5\right)$ and that due to the reorientation process $(\approx 3.9)$, found in previous investigations in the martensite state on the same specimen [10].

In the CuAlBe case, a tiny concavity can be noticed all over the ER vs. $\varepsilon$ curves, but apart from this small contribution, the equation (1) is satisfied with $\beta \approx 4.8$ ( $v$ has here been assumed equal to 0.5 , since no data are available in literature for this alloy). Unfortunately, ER measurements during the martensite reorientation process were not available in this case: the $P \rightarrow M_{n o}$. transformation and the reorientation contributions could not be evaluated separately.

As regards the NiTi specimen, a pseudoelastic behaviour is present at $79.7^{\circ} \mathrm{C}$, and equation (1) gives $\beta \approx 6$. The $\sigma-\varepsilon$ curves at 79.7 and $60.1^{\circ} \mathrm{C}$ are quite similar, but the corresponding ER vs. $\varepsilon$ curves are dif-
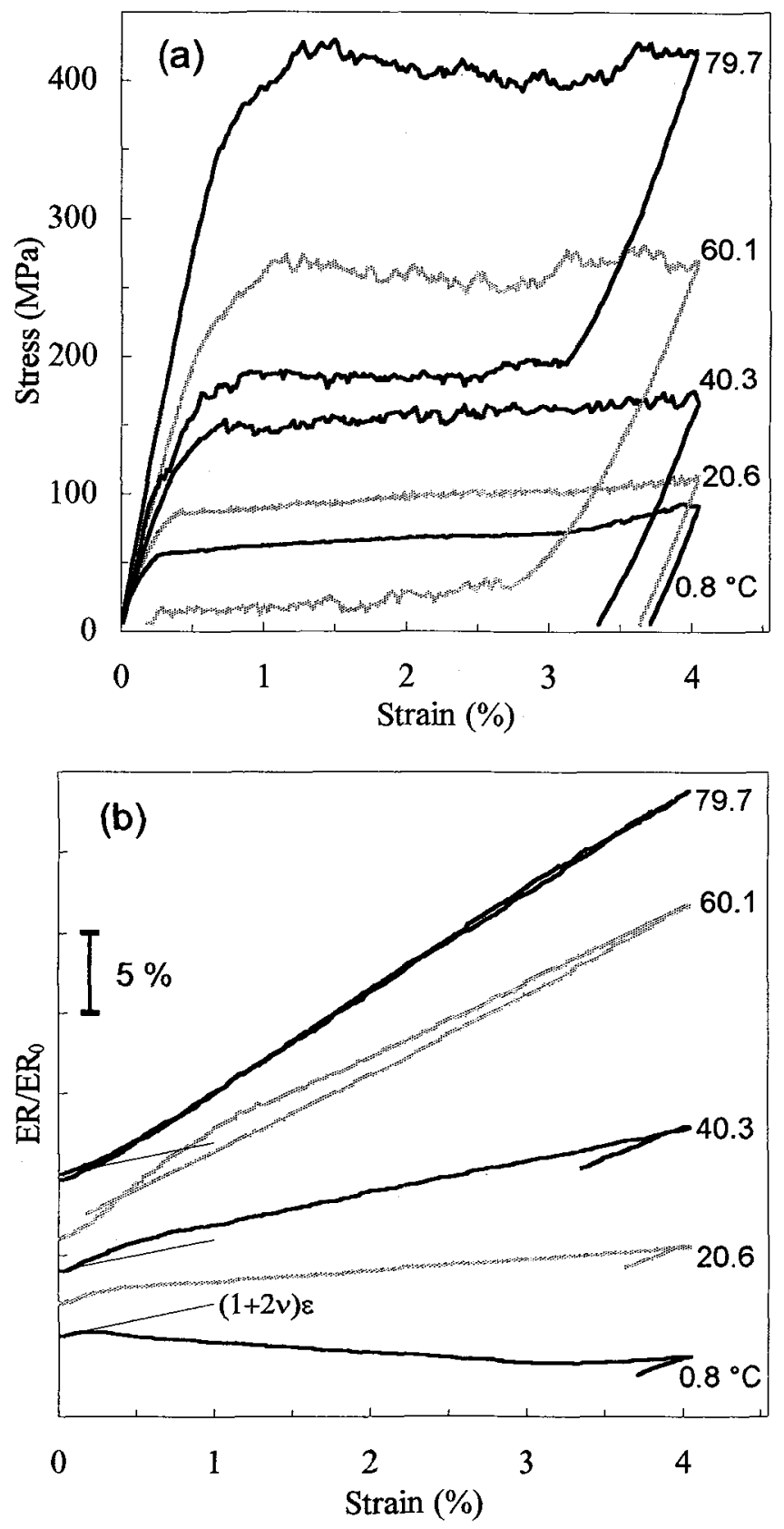

Figure 4. Tensile tests on a NiTi specimen: (a) stress vs. strain (b) ER vs. strain. The figures on the plot represent the test temperatures. For clarity, the ER curves are shifted along the $y$-axis. 
ferent. At $60.1^{\circ} \mathrm{C}$ the ER vs. $\varepsilon$ increases with a higher rate than at $79.7^{\circ} \mathrm{C}$, supporting the idea that aiso the $\mathrm{B} 2 \rightarrow$ Rphase transformation is induced for $\varepsilon<1 \%$. For larger deformation values the stress state is high enough to induce the $\mathrm{B} 2 \rightarrow \mathrm{B} 19^{\prime}$ transformation: however the rate decrease suggests that the Rphase $\rightarrow B 19$ ' transformation is also present.

The other curves show that the lower the temperature, the lower the slope: apart from an 'elastic' beginning, these curves are the result of the superimposition of the $B 2 \rightarrow B 19$ ' and the $R \rightarrow B 19^{\prime}$ ' transformations, the latter transformation prevailing on the former. Since larger Rphase initial fractions are present at lower temperatures and the $\mathrm{Rphase} \rightarrow \mathrm{B} 19^{\prime}$ transformation carries a negative electronic contribution to the ER, even a negative slope is attained at the lowest temperature.

\section{CONCLUSIONS}

The ER modification during either the thermally stable SIM transformation or in the pseudoelastic regime has been examined here in NiTi, NiTiCu and CuAlBe alloys.

In all examined alloys, in the pseudoelastic regime, in spite of different thermoelastic martensitic transformations being present, a nice linear relationship is found for ER as a function of $\varepsilon$ beyond the elastic range, confined to $\varepsilon<0.5 \%$.

The high rate of change of ER with deformation for CuAlBe, NiTi and NiTiCu alloy is 6.8, 7.7 and 10.1 respectively, only a minor part of which is due to the pure geometric contribution $(1+2 v)$.

In the pseudoelastic regime, as in the NiTiCu and $\mathrm{CuAlBe}$ alloys and in NiTi for high temperatures, a nice linear behaviour is found when only two phases are present, with a slope almost constant with temperature. But when more than two phases are present, the transformation from one kind of martensite to the other may carry a negative electronic contribution. This effect on a NiTi alloy is clearly shown in the present paper either in the pseudoelastic or the thermally stable SIM range: the Rphase $\rightarrow B 19^{\prime}$ transformation implies a negative electronic contribution which may be strong enough to overwhelm the positive geometric term.

In the light of the present results, except in the case where more than one martensitic phase is stress induced, the ER appears to be an appealing property to control deformation.

\section{Acknowledgements}

Thanks are due to Prof. A.V. Shelyakov, Moscow Eng. Physics Institute, Moscow, for supplying the NiTiCu ribbons.

The research was supported by MURST $60 \%$ and MURST $40 \%$ funds.

\section{References}

[1] Wayman C.M. and Cornelis I., Scripta metall.6 (1972) 115-122

[2] Nam T.H., Saburi T. and Shimizu K., Mater. Trans., JIM 31 (1990) 959-967

[3] Nam T.H., Saburi T. and Shimizu K., Mater. Trans., JIM 33 (1991) 814-820

[4] Lo Y.C., Wu S.K. and Horng H.E., Acta metall. mater. 41 (1993) 747-759

[5] Airoldi G., Ranucci T., Riva G. and Sciacca A., J. Phys.: Condens. Matter 7 (1995) 3709-3720

[6] Miyazaki S., Otsuka K. and Wayman C.M., ISIJ Int'l 29 (1989) 423-429

[7] Airoldi G., Ranucci T. and Riva G., J. de Physique IV-C4 (1991) 439-444

[8] Airoldi G., Ranucci T., Riva G. and Vicentini B., Mater. Res. Soc. Sym. Proc. 246 (1991) 277

[9] Matveeva N.M., Kovneristyi Yu.K., Byokovskii Yu.A., Shelyakov A.V. and Kostyanaya O.V., Russ. Metall. 4 (1989), 166-170

[10] Airoldi G., Pozzi M. and Riva G., "The Electrical Resistance Properties of Shape Memory Alloys", MRS Fall Meeting, Boston USA, 2-6 December, 1996, to be published in Proc. MRS, vol. 459 